\title{
GONIAL ANGLE DETERMINATION; A COMPARISON BETWEEN LATERAL CEPHALOGRAM AND BOTH HEMISPHERES OF ORTHOPANTOMOGRAM
}

\author{
Mehwish Khan, Faheem Nake Akhtar, Abdullah Jan, Fatima Hamid, Sana Tariq \\ Armed Forces Institute of Dentistry/National University of Medical Sciences (NUMS) Rawalpindi Pakistan
}

\begin{abstract}
Objective: To evaluate the mean values of Genial angle measured by Lateral cephalogram and Orthopantomogram. Study Design: comparative cross sectional study.

Place and Duration of Study: Armed Forces Institute of Dentistry, Rawalpindi, from Aug 2018 to Feb 2019.

Methodology: Patients irrespective of gender between the ages of 13-20 who reported for the treatment of class I malocclusion with complete anterior dentition were included. Orthopantomogram and lateral cephalogram were drawn by the same person. Gonial angle on orthopantomogram was measured by drawing tangents on posterior border of ramus and lower border of mandible on both halves of orthopantomogram. On lateral cephalogram the angle was measured between ramus and mandible. Gonial angle measured from lateral cephalogram and orthopantomogram were compared. T-test was applied.

Results: Mean age of the individuals was $18.33 \pm 3.97$ years. The mean gonial angle measured on lateral cephalogram was $122.66 \pm 1.64$ degrees while the mean gonial angle measured on right and left side of orthopantomogram was $122.41 \pm 1.56$ degrees and $122.33 \pm 1.55$ degrees respectively.

Conclusion: Orthopantomogram can also be used to measure gonial angle as accurately as a lateral cephalogram with the advantage that there are no superimpositions and both the gonial angles can be measured simultaneously on orthopantomogram.
\end{abstract}

Keywords: Gonial angle, Lateral cephalogram, Orthopantomogram.

This is an Open Access article distributed under the terms of the Creative Commons Attribution License (https://creativecommons.org/licenses/by-nc/4.0/), which permits unrestricted use, distribution, and reproduction in any medium, provided the original work is properly cited.

\section{INTRODUCTION}

Facial skeletal features are the hallmarks of identification and visualization of every individual. The mandible in this regard with its features and angulations is a prime bone of importance. One of the main specific angle which gives individualization to person is gonial angle. Gonial angle is an essential consideration of stomatognathic complex giving idea about the vertical framework and balance of maxillofacial structures ${ }^{1}$. Gonial angle can be used by the clinicians as an index of occlusal force ${ }^{2}$. Variation in gonial angle was found in age ${ }^{3}$, gender and even dental status. A study by Ceylan et al documented no significant difference among dentulous and edentulous states ${ }^{4}$. While a study by Huumonen affirmed that gonial angle was larger in edentulous subjects ${ }^{5}$. Weinmann et al concluded that progressive wasting of muscles of mastication leads to change in a mandibular angle ${ }^{6}$. Orthodontic diagnosis is derived from three main sources history, clinical examination and evaluation and analysis of dental casts, different types of radiographs and photographs ${ }^{7}$. Among radiographs lateral cephalograpm and orthopantomogram are must for every orthodontic patient. The primary aim of cephalometric analysis is

Correspondence: Dr Abdullah Jan, Orthodontics Department, Armed Forces Institute of Dentistry, Rawalpindi Pakistan

Received: 14 Oct 2019; revised received: 17 Dec 2019; accepted: 10 Jan 2020 to assess both horizontal and vertical relations of five major functional and aesthetic components of face: the cranium and cranial base, skeletal mandible, skeletal maxilla, maxillary dentition and alveolar process, mandibular dentition and alveolar process ${ }^{8}$. The vertical relationships as well as horizontal angulations, measurement and relationship are important in treatment planning and outcome determination.

Panoramic radiography was invented by Prof Yrjo Paetero of the University of Helsinki 1961. By virtue of its capacity to take a solitary depiction of whole stomatognathic system, dentition, jaws, temporomandibular joints and sinuses panoramic radiography forms a central orthodontic viewing tool. Due to intrusion of superimpositions visible on lateral cephalogram, consistent dimensions of individual mandibular angle happen to be very difficult ${ }^{9}$. One of the main advantages of orthopantomogram is that there are no super impositions ${ }^{8}$. Orthopantomogram also shows both hemispheres of mandible and both gonial angle can be compared for any underlying pathological condition like condylar hyperplasia and condylar elongation $^{10}$. Studies that observed the role of panoramic radiographs as a means of examining maxillary and mandibular relationships are lacking in orthodontic literature. However, the accuracy of gonial angle measurements from orthopantomogram need to be eval- 
uated against gonial angle measurements from lateral cephalogram. The objective of this study was to determine and assess the value of gonial angle measured on lateral cephalogram and orthopantomogram.

\section{METHODOLOGY}

This comparative cross sectional study was carried out department of Orthodontics, Armed Forces Institute of Dentistry, Rawalpindi, from August 2018 to February 2019 in. Orthopantomogram and lateral cephalogram were drawn by the same person (M.K) so as to improve reliability. Sample size was calculated using $G$ power 3.1.9.2 software, keeping the value 13 of effect size as 0.8 , alpha error as 0.05 , beta error as 0.2 , probability and power 0.8 , a sample size of 150 was calculated. Patients were selected using non probability consecutive sampling. Lateral cephalogram and orthopantomogram were collected after approval from the ethical review committee of AFID (IERB No. 905/ Trg-ABP1K2). Informed consent was taken from the patient. Lateral cephalogram and orthopantomogram were drawn on a mat acetate $0.01 \mathrm{~mm}$ tracing paper with the help of an orthodontic X-ray illuminator. Gonial angle on orthopantomogram was measured by drawing tangent on posterior border of ramus and tangent on lower border of mandible on both halves of orthopantomogram. On the lateral cephalogram angle was measured between ramus and mandible. Patients lateral cephalogram readings were placed in group A, right side of orthopentomogram reading was marked as group B and left side of orthopentomogram value was marked as group C. Patients of both genders between the ages of 13-20 who reported to orthodontic department of AFID for the treatment of class I malocclusion with complete anterior dentition were included. The subjects with a prior record of maxillofacial surgery or disorders distressing the jaw or face condition, patients taking medications like immunosuppressant and corticosteroids, subjects unable to undergo radiation exposure, patients having medical disease, skeletal disorder and metabolic disorders affecting bone turn over or with other debilitating disorders and those having history of trauma were excluded. Data was analyzed using SPSS-23. The means of gonial angle measured on lateral cephalogram and both sides of orthopantomograph are compared using t-test. Significant $p$-value was kept $\leq 0.05$.

\section{RESULTS}

Mean age of the individuals was $18.33 \pm 3.97$ years. Among 150 individuals 74 (49.3\%) were male and $76(50.7 \%)$ were female (table-I).
Mean mandibular angle measured from lateral cephalogram was $122.66 \pm 1.64$ degrees. Mean gonial angle on right side of orthopantomogram was $122.41 \pm$ 1.56 degrees and gonial angle on left side of orthopantomogram was $122.33 \pm 1.55$ degrees (table-II).

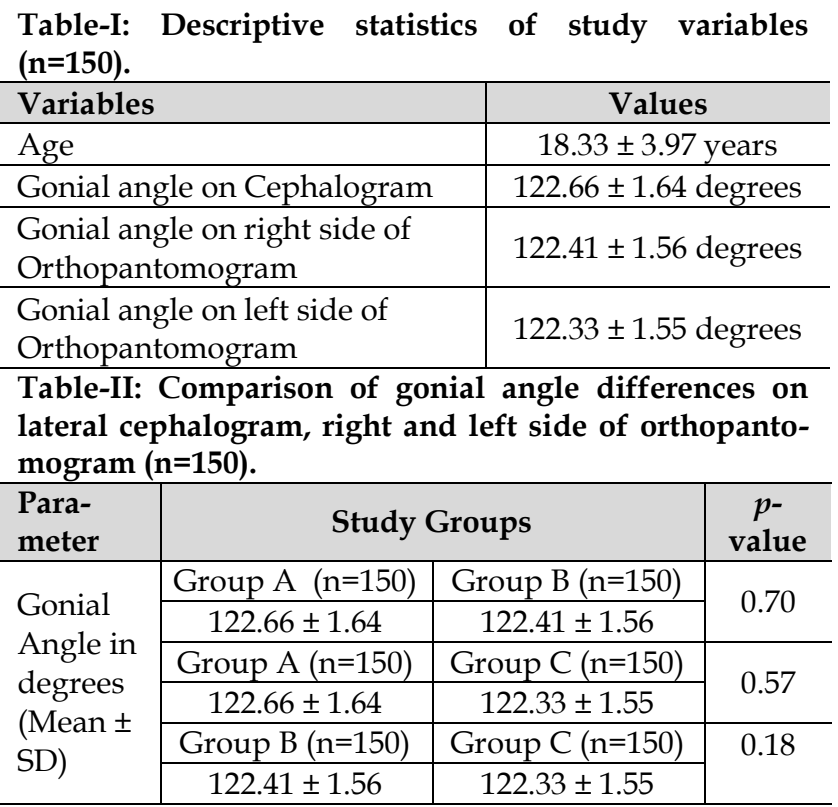

\section{DISCUSSION}

The gonial angle defines the shape and form of mandible and type of profile of patient and plays important role in determination of direction of growth pattern of specific individual. The prime objective of this research was to augment the panoramic radiographs clinical use. In orthodontic practice, panoramic radiography is advised to provide reliable diagnostic data regarding teeth growth, axial inclinations and adjacent tissues. Although there are many potential studies on panoramic radiograph emphasizing its im-portance in clinical use and highlighting its magnification and distortion problems, there are only few articles which show the importance of orthopantomograpm in describing its dento-facial and skeletal specifications and use of specific angles like gonial angle in highlighting the type of facial profile. The outcome of the study shows there is no statistically significant dissimilarity in values of gonial angle measured on panoramic radiograph and lateral cephalogram. We can assume by this study that orthopantomograph can also be used for assessment of gonial angle.

One of the main advantages of panoramic radiograph in assessing gonial angle is that both hemispheres of mandible can be viewed and measured clearly and simultaneously on a single radiograph instead of a 
single side on lateral cephalogram. This fact was established by Matilla et al11. They further determined that means of gonial angles measured on lateral cephalogram and orthopantomogram depicted that measurements from orthopantomogram are more accurate. Study conducted by Kundi was contradictory to our study showing that there was difference among the means of the gonial angles measured from lateral cephalogram and orthopantomogram ${ }^{10}$. Alhaija examined the importance of orthopantomogram to determine lower border of mandible angulation and steepness. Their study showed that gonial angle can be measured accurately from both i.e. panoramic radiograph and lateral cephalogram, also it showed that gonial angle is a potential indicator of growth direction ${ }^{12}$, this is useful because majority of oral health care professionals advice orthopantomograph during routine dental examination. It has been well documented that no significant difference is found in orthopantomogram and lateral cephalogram for gonial angle and ramus height 13. Kurt et al, use the orthopantomogram to assess asymmetry of mandible in class II subdivision malocclusion patients by measuring ramal, condylar and condylar-ramal asymmetry index and gonial angle values ${ }^{14}$. They showed that noticeable results can be found by panoramic radiograph. Shahabi et al, compared the gonial angle values in class I malocclusion obtained by orthopantomograph and lateral cephalogram ${ }^{15}$. They found no significant difference in both values concluding that panoramic radiography is as reliable as lateral cephalogram in determining gonial angle. Study conducted by Mansoor Majeed and Imtiaz Ahmed at department of orthodontics, Dow University of Health Sciences also stated that orthopantomogram can be used as substitute technique for determination of gonial angle 16. Study conducted at Khyber College of Dentistry, Peshawar also accord with our findings that orthopantomogram and lateral cephalogram can both be used to measure gonial angle with similar precision ${ }^{17}$. G Katti showed that orthopantomogram can be use accurately to measure gonial angle with the advantage that both the angles can be compared on single film without any superim positions of neighboring anatomic structures 18. Dahan et al revealed that the assessment of gonial angle basically depends upon the type of method used to measure ${ }^{19}$. The method used to measure gonial angle can either include the horizontal side of gonial angle formed by the tangent to lower border of mandible or based on a line touching gnathion point. Thus, gnathion can be easily determined on lateral cephalogram but it is difficult to identify on orthopantomo- gram so to avoid this problem the horizontal plane of gonial angle in both types of radiographs was made by a line drawn tangentially to inferior border of body of mandible. A study conducted at Ziauddin College of Dentistry by Hamamd Ul Haq concluded that panoramic radiography could be used as a reliable tool in the determination of gonial angle in different sagittal facial planes ${ }^{20}$. A study observed the association of gender, age and dental status with gonial angle through lateral cephalogram and orthopantomogram. They concluded that value of gonial angle on lateral cephalogram and orthopantomograph significantly differ. They also concluded that age and gender can be estimated more reliably through gonial angle measurements by orthopantomogram and lateral cephalograpm respectively ${ }^{21}$. Masao Araki compared the measurements of gonial angle on dry skulls derived through both lateral cephalogram and orthopantomogram ${ }^{22}$. The mean of gonial angle determined by panoramic radiography was smaller than mean of gonial angle measured on orthopantomogram but the difference was nonsignificant. This difference was attributed to head position, angle of inclination of the mandibular body and direction of $\mathrm{X}$ ray beam.

In our study the $p$-values of mean gonial angle on the right and left sides of orthopantomogram were compared with a lateral cephalogram. There was no significant disparity between values of gonial angle on orthopantomogram and lateral cephalogram. The mean value of mandibular angle determined in our study on lateral cephalogram was $122.66 \pm 1.64$ and the mean of gonial angle on right side as measured on orthopantomogram was $122.41 \pm 1.56$ and on left is 122.33 \pm 1.55 . Our $p$-value was also non-significant which showed there is no difference between the means of gonial angles measured on orthopantomogram and lateral cephalogram. With the increasing use of panoramic radiography in orthodontics, it is useful to determine gonial angles through panoramic radiographs and by doing so saving the patients extra radiation exposure and expenditure.

\section{CONCLUSION}

No statically significant difference was found between both types of radiographs (lateral cephalogram and orthopantomogram) in determination of gonial angle measurement. Predictably the orthopantomogram can also be used as a reliable diagnostic tool for determination of gonial angle measurement with the advantage that an additional $\mathrm{X}$-ray radiation and film cost is reduced, without superimpositions. 


\section{CONFLICT OF INTEREST}

This study has no conflict of interest to be declared by any author.

\section{REFERENCES}

1. Nadkerny PV, Kumar DA. Assessing reliability of mandibular planes in determining gonial angle on lateral cepha-logram and panoramic radiograph. J Orthod Res 2015; 3(1): 45-48.

2. Miwa S, Wada M, Murakami S, Suganami T, Ikebe K, Maeda Y. Gonial Angle Measured by Orthopantomography as a Predictor of Maximum Occlusal Force. J Prosthodont 2017; 28(1): 426-30.

3. Srineeraja P. Determination of angle of mandible from mandibular bones and orthopantomograph. J Pharm Sci Rev Res 2015; 7(8): 579-84.

4. Ceylan G, Yaníkoglu N, Yílmaz AB, Ceylan Y. Changes in the mandibular angle in the dentulous and edentulous states. J Prosthet Dent 1998; 80(6): 680-84.

5. Huumonen S, Sipilä K, Haikola B, Tapio M, Söderholm AL, Remes Lyly $\mathrm{T}$, et al. Influence of edentulousness on gonial angle, ramus and condylar height. J Oral Rehabi 2010; 37(1): 34-38.

6. Weinmann JP, Sicher H. Bone and Bones, Fundamentals of Bone Biology, CV Mosby Co.St. Louis 1947, [Internet] Available from: https:// www.cabdirect.org/cabdirect/abstract/19561401360

7. Radhakrishnan PD, Varma S, Kovilakam N, Ajith VV. Dilemma of gonial angle measurement: Panoramic radiograph or lateral cephalogram. Imaging Sci Den 2017; 47(2): 93-37.

8. Bhullar MK, Uppal AS, Kochhar GK, Chachra S, Kochhar AS. Comparison of gonial angle determination from cephalograms and orthopantomogram. Ind J Dent 2014; 5(3): 123-28.

9. Oh S, Kim CY, Hong J. A comparative study between data obtained from conventional lateral cephalometry and reconstructed three-dimensional computed tomography images. J Korean Assoc Oral Maxillofac Surg 2014; 40(3): 123-29.

10. Kundi I. Accuracy of assessment of gonial angle by both hemispheres of panoramic images and its comparison with lateral cephalometric radiographic measurements. J Dent Health Oral Disord Ther 2016; 4(4): 1-16.

11. Mattila K, Altonen M, Haavikko K. Determination of the gonial angle from the orthopantomogram. Angle Orthod 1977; 47(2): $107-10$.

12. Abu Alhaija ES. Panoramic radiographs: determination of mandibular steepness. J Clin Pediatr Dent 2005; 29(2): 165-66.

13. Kumar SS, Thailavathy V, Srinivasan D, Loganathan D, Yamini J. Comparison of orthopantomogram and lateral cephalogram for mandibular measurements. J Pharm Bioallied Sci 2017; 9(Suppl1): S92-95.

14. Kurt G, Uysal T, Sisman Y, Ramoglu SI. Mandibular asymmetry in Class II subdivision malocclusion. Angle Orthod 2008; 78(1): 32-37.

15. Shahabi M, Ramazanzadeh BA, Mokhber N. Comparison between the external gonial angle in panoramic radiographs and lateral cephalograms of adult patients with Class I malocclusion. J Oral Sci 2009; 51(3): 425-29.

16. Majeed M, Ahmed I. Comparison of gonial angle determination from cephalograms and orthopantomogram of patients under orthodontic treatment. J Bahria Univ Med Dent Coll 2016; 6(1): 88-91.

17. Bibi T, Rasool G. Reliability of orthopantomogram in determination of gonial angle. Pak Oral Dental J 2017; 37(2): 248-51.

18. Katti G, Katti C, Shahbaz S, Khan M, Ghali SR. Reliability of panoramic radiography in assessing gonial angle compared to lateral cephalogram in adult patients with Class I malocclusion. J Ind Acad Oral Med Radiol 2016; 28(3): 252-55.

19. Dahan J, Jesdinsky HJ. Evaluation of the orthopantomogram for cephalometric studies in orthodontics. Stoma 1968; 21(3): 200-205.

20. Mirza HH, Memon S, Agha D. Comparison of gonial angle between panoramic radiographs and lateral cephalograms in different sagittal facial patterns. Pak J Med Dent 2018; 7(2): 5-10.

21. Pillai JP, Shah RJ, Darji B, Banker A, Pillai RJ. Association of the gonial angle with age, gender, and dental status: A radiographic study using lateral cephalogram and orthopantomogram. J Forensic Radiol Imag 2018; 15(1): 8-13.

22. Araki M, Kiyosaki T, Sato M, Kohinata K, Matsumoto K, Honda $\mathrm{K}$. Comparative analysis of the gonial angle on lateral cephalometric radiographs and panoramic radiographs. J Oral Sci 2015; 57(4): 373-78. 